\title{
Light-cone gauge and the (causal) principal-value prescription
}

\author{
B. M. Pimentel and A. T. Suzuki \\ Instituto de Física Teórica, Universidade Estadual Paulista, Rua Pamplona, 145, 01405-São Paulo, SP, Brasil
}

(Received 30 March 1990)

\begin{abstract}
The principal-value (PV) prescription constrained with causality is used to treat the unphysical pole $(k \cdot n)^{-1}$ in the basic one-loop light-cone integral. It is shown that the so-modified prescription avoids the emergence of double-pole singularities, contrary to what has been previously obtained in the PV scheme without causality. However, there remains an overall factor $\frac{1}{2}$ which does not agree with the results derived in the Mandelstam-Leibbrandt (ML) prescription. This overall factor is a remnant coming from the definition of the prescription proper.
\end{abstract}

\section{INTRODUCTION}

The first time modern theoretical physicists were introduced to the light-cone formulation for quantum field theories goes as far back as 1949 , when Dirac ${ }^{1}$ discussed the front form of relativistic dynamics. For the next two decades or so since its debut, interest in this subject lay dormant, with only few sporadic appearances in the literature. (For a very nice historical survey we refer the reader to a well-written review article on noncovariant gauges by Leibbrandt. ${ }^{2}$ ) These focused not on the gauge per se but rather on investigating the structure of relativistic theories in the light-cone frame. ${ }^{3}$ It was only during the 1970's that a handful of researchers started venturing to take a fresh look at the gauge proper. ${ }^{4}$

In the beginning of the last decade, the light-cone gauge was shown to be especially appropriate for the computation of quantum effects contributing to the leading-logarithm approximation in deep-inelastic processes. ${ }^{5}$ However, as much as this feature was attractive and desirable, hand in hand with it there emerged an ominous aspect: Feynman amplitudes at the one-loop level exhibited double-pole singularities. ${ }^{6}$

Tracing back the origin of such a pathological behavior, one notes that this has frequently been ascribed to the principal-value (PV) prescription employed to treat the unphysical poles $(k \cdot n)^{-1}$ of the gauge-boson propagator, though direct application of dimensional regularization to one-loop light-cone integrals does also yield double poles. $^{7}$

Therefore, alternative prescriptions to handle the $(k \cdot n)^{-1}$ singularities in the light-cone gauge were searched for. Mandelstam ${ }^{8}$ and Leibbrandt ${ }^{9}$ authored two seemingly different prescriptions independently; later, Lee and Milgram ${ }^{10}$ showed that they were, in fact, equivalent. These new prescriptions were devised in such a way so as to ensure that the location of the $(k \cdot n)^{-1}$ poles in the $k^{0}$-complex plane would not hinder Wick rotation nor spoil power-counting thereby; qualities which, as is often claimed, were missing in the PV prescription.

Moreover, Basseto, Dalbosco, Lazzizzera, and Solda$\mathrm{ti}^{11}$ demonstrated that the Leibbrandt prescription could be recovered via canonical quantization, and more recently, within the framework of functional path integrals.
Slavnov and Frolov ${ }^{12}$ reproduced a similar result.

Successful one- and two-loop calculations employing either of those new prescriptions have already been published,,$^{13}$ enhancing their credibility as well as their suitability to handle the troublesome $(k \cdot n)^{-1}$ factors in the light-cone gauge.

So, with all this, the role of the PV prescription in this particular gauge waned, while the prescription itself was left to sink into oblivion. To add insult to injury, it went carrying an unwelcome reputation of being the prime culprit for all unwieldy pathologies manifested at its implementation.

Here we would like to take a second look at the illfavored prescription and address two questions concerning it: (a) Why is it that the PV prescription did not work in the early one-loop calculations in the light-cone gauge? (b) Is it possible to spot the cause or causes either of mathematical or physical nature that made the implementation of such a prescription result in double-pole singularities even at the one-loop level?

The answer to these questions is found in the following sections, where it is clearly shown that the deficiency is not only in the prescription per se, but also in its mistaken implementation, which used to overlook basic first principles. In other words we will understand why those unwieldy higher-order poles that plagued earlier calculations of quantum effects in this gauge are physically unacceptable.

\section{PRELIMINARIES}

At this point we introduce our notation and convention. Throughout this article, the signature for the Minkowski (flat) space-time metric is $(+,-,-,-)$ with $x^{0}$ being the time component of a general four-vector $x^{\mu}$. This is written in light-cone components as

$$
x^{\mu}=\left(x^{+}, x^{-}, x^{i}\right), \quad i=1,2,
$$

where

$$
x^{ \pm}=\frac{x^{0} \pm x^{3}}{\sqrt{2}}=\frac{x_{0} \mp x_{3}}{\sqrt{2}}=x_{\mp}
$$

and 


$$
x^{i} \equiv(\hat{x})^{i}=\left(x^{1}, x^{2}\right)
$$

It is convenient to define the lightlike vectors $n_{\mu}$ and $\bar{n}_{\mu}$ with Cartesian components

$$
n_{\mu}=\frac{1}{\sqrt{2}}(1,0,0,1)
$$

and

$$
\bar{n}_{\mu}=\frac{1}{\sqrt{2}}(1,0,0,-1) .
$$

These, therefore, satisfy

$$
n^{2}=\bar{n}^{2}=0
$$

and

$$
n^{\mu} \bar{n}_{\mu}=1
$$

while the "plus" and "minus" components are conveniently reproduced now by

$$
x^{+}=x^{\mu} n_{\mu}
$$

and

$$
x^{-}=x^{\mu} \bar{n}_{\mu} .
$$

The scalar product between two vectors becomes

$$
\begin{aligned}
x^{\mu} y_{\mu}(\equiv x \cdot y) & =x^{+} y^{-}+x^{-} y^{+}-x^{i} y^{i} \\
& =x^{+} y^{-}+x^{-} y^{+}-\hat{x} \cdot \hat{y} .
\end{aligned}
$$

In particular,

$$
x^{2}=2 x^{+} x^{-}-\hat{x}^{2} .
$$

The volume element is written

$$
d^{4} x=d^{2} \hat{x} d x^{-} d x^{+},
$$

and dimensional regularization is to be implemented by continuing the $\mu=1,2$ components to $D-2$ dimensions, i.e.,

$$
d^{D} x=d^{D-2} \hat{x} d x^{-} d x+
$$

We also employ, according to convenience, the definition

$$
D-2=2(\omega-1) \text {. }
$$

For a vector gauge field $A_{\mu}$, the light-cone gauge is defined by

$$
n^{\mu} A_{\mu}\left(\equiv n \cdot A \equiv A^{+}\right)=0, \quad n^{2}=0,
$$

where $n^{\mu}$ is an arbitrary lightlike constant vector.

Formally inverting the "regularized" quadratic form in the Lagrangian density, the propagator in momentum space reads

$$
\begin{aligned}
G_{\mu v}(k)=\frac{-i}{k^{2}+i \epsilon} & \mid g_{\mu \nu}-\frac{k_{\mu} n_{v}+k_{v} n_{\mu}}{k \cdot n} \\
& \left.+\frac{\left(\alpha k^{2}+n^{2}\right) k_{\mu} k_{v}}{(k \cdot n)^{2}}\right) .
\end{aligned}
$$

Letting the gauge parameter $\alpha$ vanish and using (2.6), the effective propagator in the light-cone gauge becomes

$G_{\mu \nu}(k)=\frac{-i}{k^{2}+i \epsilon}\left(g_{\mu \nu}-\frac{k_{\mu} n_{v}+k_{v} n_{\mu}}{k \cdot n}\right), \epsilon>0$,

where the unphysical singularity $(k \cdot n)^{-1}=\left(k^{+}\right)^{-1}$ has yet to be defined.

In what follows, our chief concern will be the evaluation of the basic one-loop light-cone integral $\left(d k \equiv d^{D} k\right)$

$$
\frac{(\mu)^{4-2 \omega}}{(2 \pi)^{2 \omega}} \int \frac{d k}{\left(k^{2}+i \epsilon\right)\left[(k-p)^{2}+i \epsilon\right] k^{+}},
$$

where $\mu$ is the mass scale produced by the renormalization procedure and $p$ is the external momentum. For convenience, we leave the factor $\mu^{4}(2 \pi \mu)^{-2 \omega}$ out and focus our attention on the integral

$$
I=\int \frac{d k}{\left(k^{2}+i \epsilon\right)\left[(k-p)^{2}+i \epsilon\right] k^{+}} .
$$

Until 1982 , the only known way to treat the pole at $k^{+}=0$ was to do it in the sense of the Cauchy principal value, i.e.,

$\frac{1}{k^{+}}=P \frac{1}{k^{+}}=\lim _{\zeta \rightarrow 0} \frac{1}{2}\left(\frac{1}{k^{+}+i \zeta}+\frac{1}{k^{+}-i \zeta}\right), \quad \zeta>0$.

Using (2.18), Capper, Dulwich, and Litvak ${ }^{14}$ arrived at the following result for (2.17):

$I=i(-\pi)^{\omega} \frac{\left(p^{2}\right)^{\omega-2}}{p^{+}} \frac{\Gamma(2-\omega) \Gamma(\omega-2) \Gamma(\omega-1)}{\Gamma(2 \omega-3)}$,

where the double pole for $\omega \rightarrow 2$ is clearly seen. This result is physically unacceptable.

On the other hand, by using the Mandelstam prescription, that is, making the substitution

$$
\frac{1}{k^{+}}=\lim _{\zeta \rightarrow 0} \frac{1}{k^{+}+i \zeta k^{-}}, \quad \zeta>0,
$$

the same authors quoted above showed that

$$
\begin{aligned}
I= & i(-\pi)^{\omega} \frac{\left(p^{2}\right)^{\omega-2}}{p^{+}} \Gamma(2-\omega) \\
& \times\left[\frac{\Gamma(\omega-2) \Gamma(\omega-1)}{\Gamma(2 \omega-3)}\right. \\
& \left.-\sum_{l=0}^{\infty} \frac{(-1)^{l} \Gamma(2-\omega+l)}{l !(\omega-2+l) \Gamma(2-\omega)}\left(\frac{\hat{p}^{2}}{p^{2}}\right)^{l}\right],
\end{aligned}
$$

which, for $\omega \rightarrow 2$ yields

$$
I=\frac{i \pi^{2}}{p^{+}}\left(\frac{\pi^{2}}{6}-S(\lambda)\right)+O(2-\omega),
$$

where $S(\lambda)$ is the Spence (or "dilogarithm") integral:

$$
S(\lambda)=-\int_{0}^{1} d x x^{-1} \ln (1-\lambda x)
$$

with

$$
\lambda=-\frac{\hat{p}^{2}}{p^{2}} .
$$


Note that (2.22) is finite, so that it is in harmony with the naive power-counting assessment done on (2.17).

From the work of Lee and Milgram, ${ }^{10}$ we know that the Leibbrandt prescription, namely,

$$
\frac{1}{k^{+}}=\lim _{\zeta \rightarrow 0} \frac{k^{-}}{k^{+} k^{-}+i \zeta}, \quad \zeta>0,
$$

when implemented in (2.17), does also yield the same result as that obtained through the Mandelstam prescription.

The status quo for the main properties of both prescriptions (2.20) and (2.25) can be summarized as follows: ${ }^{15}$ (a) Poles at $k^{+}=0$ do not hinder Wick rotation; (b) the degree of divergence of momentum integrals can be assessed by naive power counting; (c) evaluated momentum integrals exhibit divergent parts that are local functions of the external momentum; (d) there are no higher-order divergences at the one-loop level; (e) Ward and Becchi-Rouet-Stora (BRS) identities are satisfied; (f) integrals are internally consistent.

\section{THE PV PRESCRIPTION REVISITED}

Employing the standard trick of exponentiating propagators, (2.17) becomes

$$
I=-\int_{0}^{\infty} d \alpha \int_{0}^{\infty} d \beta e^{i \beta p^{2}} M_{\mathrm{LC}}(\alpha, \beta),
$$

where $M_{\mathrm{LC}}(\alpha, \beta)$ stands for the light-cone momentum integral

$$
M_{\mathrm{LC}}(\alpha, \beta) \equiv \int \frac{d k}{k^{+}} e^{i\left[(\alpha+\beta) k^{2}-2 \beta p \cdot k\right]}
$$

For convenience, we define

$$
\begin{aligned}
& x \equiv(\alpha+\beta), \\
& \beta p \equiv-x Q, \\
& \beta p^{2} \equiv x \theta .
\end{aligned}
$$

With these definitions, and using (2.10)-(2.12), the momentum integral (3.2) becomes

$$
\begin{aligned}
M_{\mathrm{LC}}(\alpha, \beta) & =\int \frac{d k}{k^{+}} e^{i x\left(k^{2}+2 k \cdot Q\right)} \\
& =\int d^{D-2} \hat{k} e^{-i x\left(\hat{k}^{2}+2 \hat{k} \cdot \hat{Q}\right)} J_{\mathrm{LC}} \\
& =\int-\left.\frac{i \pi}{x}\right|^{\omega-1} e^{i x \hat{Q}^{2}} J_{\mathrm{LC}},
\end{aligned}
$$

where the standard Gaussian integral in $(D-2)$ dimensional (recall $D=2 \omega$ ) Euclidian space has been performed and $J_{\mathrm{LC}}$ is the integral over $k^{+}$and $k^{-}$

$$
\begin{aligned}
J_{\mathrm{LC}} \equiv \int_{-\infty}^{+\infty} d k-e^{2 i x Q^{+} k^{-}} \\
\quad \times \int_{-\infty}^{+\infty} d k^{+}\left(k^{+}\right)^{-1} e^{2 i x k^{+}\left(k^{-}+Q^{-}\right)} .
\end{aligned}
$$

Let us consider the integral over $k^{+}$

$$
K_{\mathrm{LC}}=\int_{-\infty}^{+\infty} d k^{+}\left(k^{+}\right)^{-1} e^{2 i x k^{+}\left(k^{-}+Q^{-}\right)},
$$

which in the PV sense is understood as [see (2.18)]

$$
K_{\mathrm{LC}}=\frac{1}{2} \lim _{\zeta \rightarrow 0}\left[K^{+}(\zeta)+K^{-}(\zeta)\right], \quad \zeta>0,
$$

with

$$
K^{+}(\zeta) \equiv \int_{-\infty}^{+\infty} \frac{d k^{+}}{k^{+}+i \zeta} e^{2 i x k^{+}\left(k^{-}+Q^{-}\right)}
$$

and

$$
K^{-}(\zeta) \equiv \int_{-\infty}^{+\infty} \frac{d k^{+}}{k^{+}-i \zeta} e^{2 i x k^{+}\left(k^{-}+Q^{-}\right)} .
$$

We note from (3.1) and (3.3a) that $x$ is positive $(x>0)$ while the factor $\left(k^{-}+Q^{-}\right)$can either be positive or negative. Therefore, in applying the residue theorem to evaluate (3.8) and (3.9), we have the following cases to consider.

(i) $\left(k^{-}+Q^{-}\right)<0$ (or $k^{-}<-Q^{-}$). In this case, contour $C$ in the complex $k^{+}$plane must close in the lower half-plane to ensure convergence at infinity. This contour encloses the simple pole $k^{+}=-i \xi$ of (3.8), whose residue is

$$
\operatorname{Res}_{C}(-i \zeta)=e^{2 x\left(k^{-}+Q^{-}\right) \xi},
$$

so that

$$
K_{C}^{+}(\zeta)=-2 \pi i e^{2 x\left(k^{-}+Q^{-}\right) \zeta}, \quad k^{-}<-Q^{-},
$$

while the pole at $k^{+}=i \zeta$ of (3.9) is outside $C$ and its residue is zero. So

$$
K_{C}^{-}(\zeta)=0
$$

(ii) $\left(k^{-}+Q^{-}\right)>0$ (or $\left.k^{-}>-Q^{-}\right)$. For this case, contour $C^{\prime}$ must close in the upper half of the complex $k^{+}$ plane. Now the pole at $k^{+}=-i \zeta$ of (3.8) is outside $C^{\prime}$ and the corresponding residue vanishes. Therefore,

$$
K_{C^{\prime}}^{+}(\zeta)=0 \text {, }
$$

while the pole at $k^{+}=i \zeta$ of (3.9) contributes a residue of

$$
\operatorname{Res}_{C^{\prime}}(i \zeta)=e^{-2 x\left(k^{-}+Q^{-}\right) \zeta},
$$

so that

$$
K_{C^{\prime}}^{-}(\xi)=2 \pi i e^{-2 x\left(k^{-}+Q^{-}\right) \xi}, \quad k^{-}>-Q^{-} .
$$

Substituting (3.11) and (3.15) into (3.7) and inserting the result into (3.5) we obtain (the limit $\zeta \rightarrow 0$ being understood)

$$
\begin{aligned}
J_{\mathrm{LC}}=-i \pi & \left(e^{2 x \xi Q^{-}} \int_{-\infty}^{-Q^{-}} d k^{-} e^{2 i x\left(Q^{+}-i \xi\right) k^{-}}\right. \\
& \left.-e^{-2 x \zeta Q^{-}} \int_{-Q^{-}}^{+\infty} d k^{-} e^{2 i x\left(Q^{+}+i \xi\right) k^{-}}\right] .
\end{aligned}
$$

Note that the upper (lower) limit of integration over $k^{-}$in the first (second) integral of (3.16) is a direct consequence of cases (i) and (ii) considered above. Straightforward integration over $k^{-}$(with $\zeta=0$ ) and further integrations over $x$ and $y$ according to the standard change of variables 


$$
\begin{aligned}
& \alpha=x y, \\
& \beta=x(1-y)
\end{aligned}
$$

[with (3.3a) satisfied], will lead us to the physically unacceptable result (2.19).

Here is our crucial argument: We cannot and must not overlook the pole generated by the covariant piece of the propagator, namely, $k^{2}+i \epsilon=0, \epsilon>0$, which, using (2.11) reads

$$
k^{2}+i \epsilon=2 k^{+} k^{-}-\hat{k}^{2}+i \epsilon=0, \quad \epsilon>0 .
$$

This shows that there is a simple pole at

$$
k^{+}=\frac{\hat{k}^{2}}{2 k^{-}}-\frac{i \epsilon}{k^{-}}, \epsilon>0 .
$$

Whatever the value of $\hat{k}^{2}$ (in particular $\hat{k}^{2}=0$ ), the sign of the imaginary part of (3.19) must agree with the sign of the poles in prescription (2.18), otherwise causality of the whole propagator would be in jeopardy, i.e., we must have, in Eq. (3.8),

$$
\zeta=\frac{\epsilon}{2 k^{-}}, \quad \zeta>0, \epsilon>0
$$

while in (3.9),

$$
\zeta=-\frac{\epsilon}{2 k^{-}}, \quad \zeta>0, \epsilon>0
$$

Since $\zeta$ and $\epsilon$ are both strictly positive, (3.20) obliges us to take only $k^{-}>0$; conversely, (3.21) entails us to take only $k^{-}<0$. As they stand in (3.16), both integrals over $k^{-}$inadvertently allow for forbidden regions of $k^{-}$. They include those regions of $k^{-}$which forces a change in the sign of $\epsilon$ in (3.20) and in (3.21), thus mixing positive-frequency (or -energy) radiation propagating forward in time, with negative-frequency radiation. This violates causality, a basic physical principle.

Therefore, physically acceptable regions of integration over $k^{-}$(those which preserve causality) are confined to the following intervals. In (3.11),

$$
0<k^{-}<-Q^{-}
$$

while in (3.15),

$$
-Q^{-}<k^{-}<0 \text {, }
$$

so that

$$
\begin{aligned}
J_{\mathrm{LC}}= & \frac{1}{2} \lim _{\zeta \rightarrow 0}(-2 i \pi) \\
\times & {\left[\Theta\left(-Q^{-}\right) e^{2 \times \xi Q^{-}} \int_{0}^{-Q^{-}} d k^{-} e^{2 i x\left(Q^{+}-i \xi\right) k^{-}}\right.} \\
& \left.-\Theta\left(Q^{-}\right) e^{-2 \times \zeta Q^{-}} \int_{-Q^{-}}^{0} d k^{-} e^{2 i x\left(Q^{+}+i \xi\right) k^{-}}\right],
\end{aligned}
$$

where the $\Theta\left(\mp Q^{-}\right)$are the usual step functions.

Carrying out the $k^{-}$integration and taking the limit $\zeta \rightarrow 0$ we obtain

$$
\begin{aligned}
J_{\mathrm{LC}} & =-\frac{\pi\left(e^{-2 i x Q^{+} Q^{-}}-1\right)}{2 x Q^{+}}\left[\Theta\left(-Q^{-}\right)+\Theta\left(Q^{-}\right)\right] \\
& =-\frac{\pi\left(e^{\left.-2 i x Q^{+} Q^{-}-1\right)}\right.}{2 x Q^{+}}
\end{aligned}
$$

which inserted into (3.4) results in

$$
M_{\mathrm{LC}}=-\frac{1}{2}(-i)^{\omega-1} \frac{\pi^{\omega}}{x^{\omega} Q^{+}}\left(e^{-i x Q^{2}}-e^{i x \hat{Q}^{2}}\right) .
$$

This result is exactly half of that obtained through using the Mandelstam-Leibbrandt (ML) prescription. We are thankful to W. Kummer for pointing this out to us. ${ }^{16}$

Finally, changing variables according to (3.17a) and (3.17b) and integrating over $x \quad(0<x<\infty)$ and $y$ $(0<y<1)$, we arrive at a result which is half the one quoted in (2.21). This overall factor $\frac{1}{2}$ ) is a remnant from the definition of PV prescription given in (2.18).

\section{COMMENTS AND CONCLUSIONS}

By looking at the pole in the $k^{+}$complex plane generated by the covariant piece of the gauge-boson propagator and noting that the sign of its imaginary part must be concordant with the signs of the corresponding poles at $k^{+}=0$ in the sense of Cauchy PV, we observed that (i) the constraint defines a finite range of integration over the $k^{-}$integrals, ${ }^{17}$ and (ii) the basis for this restriction has to do with ensuing that positive-energy quanta do not become mixed up with quanta of negative energy propagating forward in time. The point is that the propagator as a whole must be thus causal.

One should note that the poles of prescription (2.18) in the $k^{0}$-complex plane, when analyzed under observation (ii) above, are located in the second and fourth quadrants. This ensures legitimacy to Wick rotation and thus for power counting.

We want to emphasize that careful implementation of the PV prescription in the light-cone gauge reproduces (apart from an overall factor $\frac{1}{2}$ ) the well-behaved properties ensuing from treating the $(k \cdot n)^{-1}$ poles via either the Mandelstam or the Leibbrandt prescriptions. It should be stressed that earlier attempts at applying the PV technique in the light-cone gauge computations failed because they overlooked the fact that there is a strong constraint over the range of the $k^{-}$component of momentum. Overlooking this amounts to violating causality, and so, there is little wonder unwieldy double poles in one-loop calculations appeared. However, the PV prescription per se entails an overall factor $\frac{1}{2}$ which renders it unsuitable for handling light-cone gauge singularities.

Let us now double back and retrace the crucial arguments that entered our calculation. We started off from the standard definition for the PV prescription; then brought in the requirement entailed by causality of the whole propagator (in passing we point out the fact that it is only when this step is correctly taken into account that the evaluation of the $k^{-}$integral does converge for $x \rightarrow \infty)$, and then arrived at a result which equals half of that obtained through using the M-L prescription. This 
strongly suggests that causality plays a key role in the whole calculation and that causality itself must be the guiding factor to treat both the covariant-type pole $\left(q^{2}\right)^{-1}$ and the so-called "spurious" or "unphysical" poles of the type $(q \cdot n)^{-n}, n=1,2, \ldots$

A preliminary calculation carried out in the pure axial gauge does also corroborate this conclusion, the results of which will be published shortly elsewhere.

Of course, we have only evaluated the basic one-loop integral in the light-cone gauge, (2.17). However, in computing, e.g., the one-loop gluon self-energy, one encounters tadpolelike integrals as well as integrals with a tensorial structure in the integrand. These can be evalu- ated following the same steps as the ones used for evaluating (2.17), or by employing techniques already developed for the Mandelstam and/or Leibbrandt prescriptions to compute those kinds of integral in terms of the basic one (2.17).

\section{ACKNOWLEDGMENTS}

A.T.S. wishes to thank the kind hospitality of Instituto the Fisica Teórica. B.M.P. was partially supported by and A.T.S. was supported by Conselho Nacional de Desenvolvimento Científico e Tecnológico, Brazil.
${ }^{1}$ P. A. M. Dirac, Rev. Mod. Phys. 21, 392 (1949).

${ }^{2}$ G. Leibbrandt, Rev. Mod. Phys. 59, 1067 (1987).

${ }^{3}$ S. Fubini and G. Furlan, Physics 1, 229 (1965); L. Susskind, Phys. Rev. 165, 1535 (1968); K. Bardackci and M. B. Halpern, ibid. 176, 1686 (1968); S-J. Chang and S-K. Ma, ibid. 180, 1506 (1969).

${ }^{4}$ A. Chakrabarti and C. Darzens, Phys. Rev. D 9, 2484 (1974); D. J. Gross and F. Wilczek, ibid. 9, 980 (1974); J. M. Cornwall, ibid. 10, 500 (1974).

${ }^{5}$ D. J. Pritchard and W. J. Stirling, Nucl. Phys. B165, 237 (1980); G. Curci, W. Furmanski, and R. Petronzio, ibid. B175, 27 (1980).

${ }^{6}$ See, for example, Curci, Furmanski, and Petronzio (Ref. 5).

${ }^{7}$ See, Chakrabarti and Darzens (Ref. 4), and Pritchard and Stirling (Ref. 5).

${ }^{8}$ S. Mandelstam, Nucl. Phys. B123, 149 (1983).

${ }^{9}$ G. Leibbrandt, Phys. Rev. D 29, 1699 (1984).

${ }^{10}$ H. C. Lee and M. S. Milgram, Nucl. Phys. B268, 543 (1986).
${ }^{11}$ A. Bassetto, M. Dalbosco, I. Lazzizzera, and R. Soldati, Phys. Rev. D 31, 2012 (1985).

${ }^{12}$ A. A. Slavnov and S. A. Frolov, Teor. Mat. Fiz. 73, 199 (1987).

${ }^{13}$ M. Dalbosco, Phys. Lett. 163B, 181 (1985); D. M. Capper, D. R. T. Jones, and A. T. Suzuki, Z. Phys. C 29, 585 (1985); A. Smith, Nucl. Phys. B261, 285 (1985); B267, 277 (1986); D. M. Capper, D. R. T. Jones, and M. J. Litvak, Z. Phys. C 32, 221 (1986); H. C. Lee, M. S. Milgram, and A. Andrasi, ibid. 33, 107 (1986); G. Leibbrandt and S-L. Nyeo, J. Math. Phys. 27, 627 (1986); H. C. Lee, and M. S. Milgram, Nucl. Phys. B268, 543 (1986); A. T. Suzuki, Z. Phys. C 38, 595 (1988).

${ }^{14}$ D. M. Capper, J. J. Dulwich, and M. J. Litvak, Nucl. Phys. B241, 463 (1984).

${ }^{15}$ G. Leibbrandt and T. Matsuki, Phys. Rev. D 31, 934 (1985).

${ }^{16} \mathrm{~W}$. Kummer (private communication).

${ }^{17}$ See S-J. Chang and S-K. Ma (Ref. 3). 